\title{
La Responsabilidad Social o sostenibilidad: un enfoque desde el entorno y la comunicación
}

\section{Social Responsibility or sustainability: an approach from the environment and communication}

Dra. Cecilia Burgos Romero es periodista e investigadora independiente (Chile) (burgosceci@gmail.com) (https://orcid.org/0000-0002-5084-9868)

\begin{abstract}
Resumen
El presente trabajo tiene como objetivo mostrar los diversos aportes que se han dado en el ámbito académico, especialmente desde las áreas de Economía y Administración Empresarial, sobre la Responsabilidad Social -RS-y mostrar los diferentes entornos que resultan necesarios considerar cuando ésta se estudia. Se pretende de esta manera entregar una visión sobre la RS desde una perspectiva interdisciplinaria, basándose en algunas de las definiciones que se han dado sobre esta teoría y contrastando los diversos aportes. Además, se analizan los conceptos de Entorno, Cultura Empresarial y Comunicación Organizacional, Institucional o Corporativa, por cuanto se plantea que la Comunicación está directamente relacionada con la RS, por lo que debiera ser considerada en todos los ámbitos de la actividad humana. Los análisis, resultados y sugerencias que aquí se exponen corresponden a una investigación de carácter exploratorio y bibliográfico, que se inició de manera independiente a finales de la década de los ochenta. Los resultados obtenidos señalan que la RS o Sostenibilidad, a pesar que su estudio a nivel académico data de hace más de tres décadas, sólo en los últimos 10 años en Chile ha tenido visibilidad pública, de manera especial en los medios de comunicación convencionales (radio, prensa y TV). Al mismo tiempo, se concluye que la Comunicación Interna y la Información no son consideradas como una de las responsabilidades básicas de toda empresa u organización.
\end{abstract}

\begin{abstract}
The purpose of this paper is to show the numerous academic contributions in the areas of Economy and Business Management about the Social Responsibility Theory, and to show the different settings or scenarios which are important to consider when this topic is studied. The main objective is to provide a view of Social Responsibility from an interdisciplinary perspective, supporting it with definitions of Social Responsibility Theory and contrasting different contributions. There is an analysis of the following concepts: Environment, Corporate Culture and Organizational Communication. The article states that Communication is directly related to $S R$, reason for which it should be considered in every aspect of human activity. The analysis, results, and suggestions presented are part of an exploratory and bibliographic research, which started at the end of the 1980s. The results obtained suggest that, although the subject of SR or Social Sustainability has been studied for more than three decades, it has only received public attention in the last 10 years, mainly in traditional media such as Radio, Press and TV. It is concluded that companies or corporations do not consider Internal Communication and Information as one of their basic responsibilities.
\end{abstract}

\section{Palabras clave I keywords}

Responsabilidad Social, sostenibilidad, entorno, comunicación, comunicación digital, cultura organizacional. Social Responsibility, Sustainability, environment, communication, digital communication, organizational culture.

Cómo citar: Burgos Romero, C. (2018). La Responsabilidad Social o sostenibilidad: Un enfoque desde el entorno y la Comunicación. Retos Revista de Ciencias de la Administración y Economía, 8(16), 47-60. https://doi.org/10.17163/ret.n16.2018.04 


\section{Introducción}

A más de una década de iniciado el siglo XXI y a cerca de treinta años del comienzo de las investigaciones en el ámbito académico sobre la Responsabilidad Social -RS-, resultan paradójicas las cifras que señalan que sólo un 22\% de las 100 multinacionales más grandes del mundo, han progresado en incorporar la RS en sus operaciones diarias (Rochlin, 2005).

Para los profesionales de las Ciencias Económicas y Administrativas -ingenieros comerciales, economistas, administradores de empresa- y para las propias empresas, esta situación es un misterio «¿cómo lograr que la Responsabilidad Social Corporativa -RSC- sea un elemento constituyente de la estrategia y filosofía de negocios de una empresa?», se pregunta Steve Rochlin en la publicación citada (2005).

Sería ambicioso señalar que en este trabajo se dará la respuesta a esa interrogante, pero sí se puede afirmar, con la modestia que debe guiar toda investigación, que con una visión crítica y desde la perspectiva comunicacional, se espera contribuir a que la RS sea comprendida, aplicada, considerada y difundida como una herramienta valiosa para la empresa y necesaria en toda actividad humana, no importando de qué tipo de organización o grupo se trate, como tampoco, del tamaño físico y reputacional de la institución. En definitiva, se postula que la RS debe estar intrínsecamente relacionada con la especie humana.

Desde la década de los 80 , en que comenzaron a realizarse los estudios sobre la «Teoría de la Responsabilidad Social», como la denominaron sus creadores, esta línea de análisis teórico-práctico ha recibido varios nombres o más bien se le han ido agregando «segundos apellidos». Así tenemos hoy, Responsabilidad Social Empresarial -RSE-, Responsabilidad Social Corporativa -RSC-, Responsabilidad Social Universitaria -RSU- y aquí se esboza el planteamiento una Responsabilidad Social Individual.

Este análisis se hace sobre la RS, así, sin apellidos, incluso sin el último nombre -RS Individual-, que se menciona en el párrafo precedente y que podría llevar a la confusión, cuando se afirma que la Responsabilidad Social debe estar intrínsecamente relacionada con la especie humana.

En este artículo, además de la RS se analiza el entorno y la comunicación, y para ello se consideran los aportes que entrega la Cultura Corporativa.

\section{Material y fuentes de información}

\subsection{RS: ¿Una o varias responsabilidades?}

$\mathrm{Al}$ igual como existen variadas definiciones de RS, también se han ido dando distintos nombres a esta «Teoría de la Responsabilidad Social», que tiene entre sus más destacados ideólogos a Kenneth Andrews, quien junto a Ulrich Beck y otros, son considerados autoridades en esta materia. Andrews (1977) al referirse a la RS puntualiza que:

[...] se trata de demandas que las empresas deben atender en razón a ser parte de la sociedad, la cual puede exigirles cosas que le preocupan e interesan, las que son cambiantes a través del tiempo; frente a esto la Empresa debe actuar de manera activa en los 
asuntos públicos y también en adquirir responsabilidades por el impacto de su actividad económica en la sociedad (p.123).

A pesar de las múltiples definiciones de RS, todas ellas tienen en común el plantear que es un quehacer que incorpora:

- Respeto por los valores éticos,

- Respeto por las personas,

- Respeto por las comunidades y

- $\quad$ Respeto por el medio ambiente.

En algunas de las definiciones de la RS, se la presenta con una directa relación con la Ética, por ejemplo, en una de ellas se señala que:

Ser socialmente responsable implica asumir, significa reconocer y aceptar los resultados sociales derivados del propio y libre operar, y por ende, hacerse cargo de las injusticias y faltas existentes en la sociedad y cuya solución está en nuestras manos. De este modo, una organización socialmente responsable asegura su sustentabilidad en el largo plazo, ya que asegura el entorno del cual depende para funcionar (Blog de Ética, 2011, s/p).

Lo expresado ut supra muestra a la RS como el compromiso contraído por las acciones $\mathrm{u}$ omisiones de cualquier individuo o grupo, que generen un impacto en la sociedad, pudiendo recaer éstas en una persona, organización, gobierno o empresa. Además, esas acciones suelen traer consigo una valoración positiva o negativa por parte de la comunidad, es decir, desde el punto de vista comunicacional podemos hablar de imagen y de reputación, negativa o positiva.

Siguiendo con el análisis que allí se plantea, se expresa que es posible detectar diversos tipos de acciones de responsabilidad social, por ejemplo, gubernamentales -como el «Bicing» o transporte urbano basado en el uso compartido de la bicicleta desarrollado en Barcelona, España (Bicing, 2018)-; de instituciones no gubernamentales, cuya esencia misma es socialmente responsable, como es el caso de Greenpeace; e incluso se agregan acciones de responsabilidad social personal o individual, pero ésta última no está suficientemente fundamentada y, en general, se la relaciona directamente con actos de filantropía o claramente de acciones para mantener o mejorar la reputación personal, a manera de ejemplo, las nominaciones que hace UNESCO y UNICEF de «Embajadores de Buena Voluntad», por la que se designan a personas reconocidas en su ámbito laboral, como futbolistas y actrices, para que difundan el quehacer que llevan a cabo esos organismos en los distintos países.

Este enfoque, aunque no está directamente relacionado con la RS, sirve para ilustrar la necesidad de contemplar y valorar la Responsabilidad Social, desde una perspectiva amplia y personal. Para ello se rescata la afirmación, considerada como la filosofía de la Responsabilidad Social, y es que «no se puede estar inserto en este mundo y permanecer indiferente ante sus problemas».

Para comprender con mayor claridad esta temática, debemos compartir el significado del valor Responsabilidad, como una actitud consciente asumida por la persona, que la compromete ante determinado hecho o acción realizada. Desde esta perspectiva, la Responsabilidad se puede analizar en dos vertientes: 
Figura 1. Tipos de adopción de la Responsabilidad

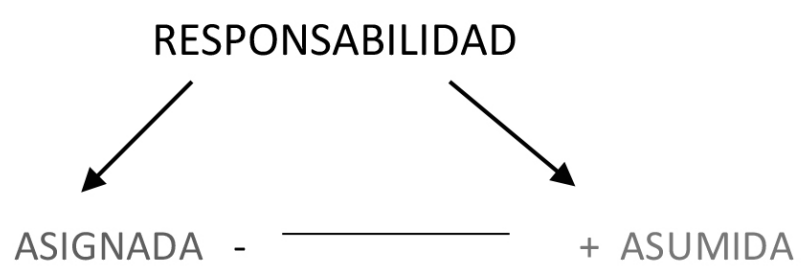

Conciencia

(Ésta se refiere a la que es impuesta u ordenada)
(La que adopta como compromiso personal)

Fuente: Blog de Ética, 2011, s/p.

Como se aprecia en la figura precedente, la responsabilidad asignada es la impuesta y se refiere a ésta cuando se le asigna -obliga- a una persona u organización, a través de las autoridades y de los parámetros establecidos (leyes, normativas específicas), sin que exista un previo análisis y toma de conciencia por parte del individuo o institución. Aunque no se niega que en esta vertiente exista conciencia, no es lo usual ya que no se asume, sino que se impone.

La otra fuente de responsabilidad es la asumida. En ésta las organizaciones y las personas la adoptan - de manera voluntaria- a partir de sus valores, creencias, ideas y la concientizan con mayor profundidad. Este tipo de responsabilidad es la que más perdura en el tiempo y la que logra un mayor impacto positivo en la sociedad. Por tanto, es la que hay que potenciar y fomentar en aras de alcanzar una mejor calidad de vida.

Por lo anterior se puede colegir que la RS es una actitud voluntaria asumida por las diferentes personas u organizaciones en aras de mejorar y satisfacer las necesidades del entorno en donde esté enclavado en un momento dado.

En la introducción del presente artículo se dieron algunos de los nombres asignados a la RS. Aquí se agrega el de «RES»-Responsabilidad de la Empresa en la Sociedad-, designación que se otorga en Argentina a esta Teoría (Paladino et al., 2006). Es necesario detenerse un segundo en este punto, porque, al igual como plantean los autores, que lo que ocurre en el empresariado trasandino, quizás también es lo que se presenta en otros países. Nos referimos, al temor o abierto rechazo que provoca en algunos empresarios el vocablo «social». Según Paladino et al. (2006) «el término RSE confunde a los empresarios ya que lo asocian inmediatamente con la necesidad de inversión social».

Otro de los vocablos, para referirse a la RS es el de «Reputación Corporativa» -RC-, acuñado por Villafañe (2003), aunque el precitado autor no se refiera a la Responsabilidad Corporativa -RC-, aunque compartan los mismos acrónimos. No hay que confundirlos, pues la Reputación Corporativa de Villafañe tiene cierta relación con la RSE, por cuanto plantea que la reputación de una compañía se logra por varias medidas, tanto internas como externas, que debe asumir la empresa con respecto a la sociedad en donde se desarrolla. 
Prosiguiendo con las distintas denominaciones que se dan a la RSE se agregan las de Responsabilidad Social Corporativa -RSC-, enfoque que también suele ser conocido como "Ciudadanía Corporativa». The Center for Corporate Citizenship, del Boston College, define "Ciudadanía Corporativa» como la manera en que la empresa integra valores sociales básicos en sus prácticas comerciales, operaciones y políticas cotidianas. Según el Banco Mundial, la «Ciudadanía Corporativa» se basa en el reconocimiento de que las empresas tienen derechos y responsabilidades que van más allá de la maximización de las ganancias en el corto plazo (Navarro, 2012, p. 79).

En lo referente a RSC es necesario mencionar a Porter, estudioso del tema de la Responsabilidad Social, quien en sus libros y artículos hace uso de la expresión «Responsabilidad Social Corporativa» y los alcances que plantea son valederos para la responsabilidad en general, es decir, con cualquiera de los nombres que se la quiera presentar.

Porter \& Kramer (2007) indican que la mayoría de los enfoques que se están haciendo sobre este tema, la RSC o RSE, ponen a la sociedad y a la corporación o empresa, enfrentándose, como enemigos, siendo que la realidad y el futuro de ello es y debe ser distinto, además que ambos son interdependientes. Sobre este casi «enfrentamiento», señala Porter \& Kramer (2007) «Gobiernos, activistas y medios de comunicación se han vuelto adeptos a perseguir rendición de cuentas de parte de las empresas por las consecuencias sociales de sus actividades» (pp. 24-38).

$\mathrm{Al}$ respecto, los autores referenciados dan varios ejemplos, como el caso de Nike, denunciado por The New York Times por prácticas laborales abusivas con sus proveedores indonésicos. Sobre este punto es pertinente agregar que lamentablemente este tipo de situaciones han seguido produciéndose, a pesar de las denuncias que mantienen de forma continua los medios de comunicación.

Es oportuno agregar otros valiosos aportes respecto a la RS o RSE, como son los de Sen -Premio Nobel de Economía-. Sen se refiere a la responsabilidad, pero pone el acento en la «Ética del Desarrollo» y Kliksberg profundiza en la «Gerencia Social» o «Capital Social» (Sen \& Kliksberg, 2007). Ambas líneas de análisis tienen como punto de partida la persona y el actuar ético, y aunque no hacen uso de la expresión RS, sus propuestas están directamente relacionadas con esta teoría y es por ello que se las menciona.

Además, es necesario considerar el concepto de «Trabajo Decente», propuesto por la Organización Internacional del Trabajo (OIT) que establece las características que debe tener una relación laboral que cumpla con los estándares laborales internacionales, de manera que el trabajo se realice en condiciones de libertad, igualdad, seguridad y dignidad humana. Por debajo de esos estándares debe considerarse que se han violado los Derechos Humanos del trabajador afectado, y que no existe trabajo libre.

En total contraposición a los autores señalados está Friedman (1975), quien no niega la existencia de las responsabilidades sociales, pero señala que quien debe dar respuesta a ellas no es la empresa, sino los individuos de forma particular. Su planteamiento se contrapone a la mayoría de los economistas de los siglos XX y XXI. La tendencia friedmaniana ha sido denominada «Teoría de los accionistas» o «Teoría de Optimización o maximización de Beneficios». Friedman, tiene entre sus seguidores a Rostow, quien postula que «la RS es de los individuos y no de las entidades, las cuales 
sólo deben atender el cumplimiento de sus finalidades específicas: obtener el mayor beneficio posible para sus accionistas» (en Paladino et al., 2006, p. 28).

Incluso al referirse a daños masivos a la población -como es la polución- expresan que el daño del ambiente se mantendrá en tanto no afecte a los beneficios, pero a algo se llegaría para atenderla si apareciera un boicot a los productos de la empresa. Sobre este punto, los comentarios están demás. La propia afirmación se auto-elimina, más aún en el momento actual, en que temas como la huella de carbono, calentamiento global, y la capa de ozono, por mencionar algunos, son tópicos de interés y preocupación a escala global.

En definitiva, frente a la interrogante ¿Una o varias Responsabilidades Sociales?, se concluye que sólo existe una, la cual ha sido enriquecida y también adaptada a los diversos campos en que ella existe o debiera estar presente. La Teoría de la RS, iniciada en el campo de investigación de las Ciencias Económicas ha recibido aportes de otras disciplinas, como la Filosofía, Sociología, Psicología y Ciencia de la Comunicación, entre otras. Es por ello que todo estudio en este ámbito debe ser de carácter transdisciplinario. Al respecto, en el presente artículo se plantea que la Ciencia de la Comunicación podría hacer grandes aportes, más aún en la Era de la Información y más recientemente Era del Conocimiento.

\subsection{Entorno}

Relacionado directamente con la RS, se encuentra el «Entorno», porque es en un escenario, real o virtual, material o humano, interno o externo; en donde se desarrolla la Responsabilidad Social. El vocablo «entorno», a pesar de ser una palabra de uso habitual en nuestro lenguaje, desde hace más de tres décadas comenzó a ser una expresión analizada en diversos ámbitos, como por ejemplo, en la Comunicación y en la Economía.

Al igual como se señaló en las primeras investigaciones en el campo de la Comunicación que el público al cual iban dirigidos los mensajes de los medios de Comunicación tradicionales tenía las características de ser amplio, heterogéneo y anónimo, en la actualidad respecto al «entorno» éste también resulta ser amplio, heterogéneo y lamentablemente muchas veces anónimo para las organizaciones.

Sobre el particular Capriotti (2009) hace un detallado análisis y clasificación de «entorno» y entrega una definición de Robbins, que lo explica como «las instituciones o fuerzas que afectan la actuación de la organización, y sobre las cuales ésta tiene muy poco o nulo control» (pp.161-168). El autor divide al «entorno» en general y específico. El general está compuesto por los factores: político-legal, económico, sociocultural y tecnológico. Por su parte, el entorno específico, puede dividirse en entorno competitivo y entorno de trabajo.

En este punto es imprescindible considerar lo planteado por Porter $(1991 ; 2007)$, quien se refiere al «Entorno Nacional» como aquel que juega un papel importante en el éxito competitivo de las empresas. Su planteamiento es holístico y en él confluyen todas las variables que se pueden detectar en una organización, entre ellas está la formación o preparación de los directivos y empleados de una empresa.

Porter (1991; 2007), afirma que el éxito de las empresas es resultante de contar con equipos de profesionales idóneos dentro del área de su competencia, por ejemplo, los muebles de Dinamarca son reconocidos mundialmente y ello se debe a que tienen 
diseñadores de gran calidad y de formación universitaria. Similar realidad es la de Japón en lo que respecta a la electrónica y la computación, pues Japón cuenta con un número de licenciados en ingeniería, per cápita, muy superior al de casi todas las demás naciones. Esto forma parte de los «factores avanzados», como Porter (1991; 2007) denomina a aquellas características que hacen que una nación sea más competitiva en determinada materia.

$\mathrm{Al}$ analizar el «Entorno», es necesario considerar la relación que tiene con él la institución o empresa, y esta aproximación puede ser de manera directa, indirecta o virtual, partiendo de ello es que se establece una diferenciación entre entornos mediatos, inmediatos y virtuales.

Antes de especificar en qué consisten estos tres tipos de relación, se define «Entorno» como el conjunto variado e indeterminado de personas, instituciones y normas, que directa o indirectamente están en relación con la organización -empresa-, y cuyo quehacer o sólo presencia, afecta a ese otro conjunto de elementos o ambiente que la rodean. Por tanto, el «Entorno Inmediato» es el conjunto variado e de personas, instituciones y normas, que están en directa relación con la organización -empresa-; el «Entorno Mediato» es aquel que tiene un cierto grado de relación con la empresa y en muchos casos ninguno, y el «Entorno Virtual» es el conjunto variado de públicos, amplios, heterogéneos y anónimos, que se encuentran en relación virtual, es decir, bajo el soporte de las TIC. Este entorno resulta difícil de delimitar o enumerar, pero no debe ser ignorado. El «Entorno Virtual» nos es impuesto de manera natural por el desarrollo que han tenido las Tecnologías de la Información y la Comunicación (TIC). Extendiendo esta clasificación de entornos a la RS, tenemos la RS Interna, hacia el interior de la organización, y la RS Externa, todo lo que involucra lo exterior a la institución.

Figura 2. Clasificación de entornos de Empresa (E)

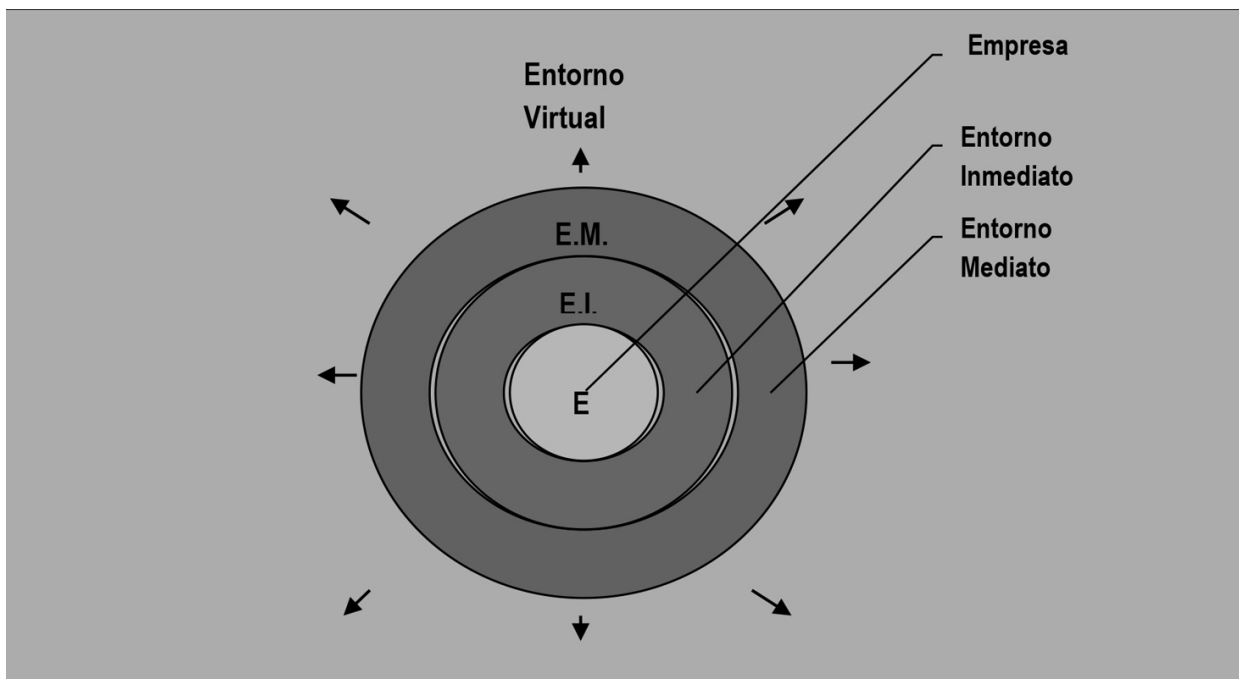

Nota: Entorno Inmediato (E.I.), Entorno Mediato (E.M.) y Entorno Virtual (E.V.) Fuente: Elaboración propia 


\subsection{Cultura Empresarial / Cultura Organizacional}

Se considera necesario hablar de cultura, o más bien, de cultura empresarial, cuando se analizan temáticas tales como la organización, el cumplimiento o visión que se tiene de la RS, su entorno y el papel que juega la comunicación en este amplio campo de estudio. A pesar, que se puede deducir que la Cultura Empresarial existe más allá de las definiciones, es necesario entregar una que facilite el compartir con mayor claridad a qué nos referimos cuando hablamos de ello.

La Cultura Empresarial es el conjunto de normas, escritas o no, que componen la base del comportamiento de los empleados de la compañía, y que inspiran tanto sus relaciones internas, como las que mantienen con sus diferentes públicos, desde los clientes a la sociedad en general (Bell Mallen, 2004).

Esta definición, como su nombre lo indica, se aplica a la RSE -Responsabilidad Social Empresarial-, pero se puede hacer extensiva a todo tipo de organización social, para ello es necesario cambiar las expresiones «empresarial», por «organizacional», «compañía», por «institución» u «organización» y sacar la palabra «clientes» por «stakeholders» o "grupos de interés». De esta forma podemos referirnos a la Cultura Organizacional de todo grupo humano, que va desde los integrantes o trabajadores de una empresa, un Ministerio, una ONG o de un club deportivo.

Por cuanto en todo grupo humano organizado bajo un mismo objetivo es necesario conocer su cultura, ese conocimiento permitirá reflejar su imagen, conocerla y mejorarla. Esto último desde el punto de vista investigativo de la comunicología, es decir, desde la teoría y de la práctica en el campo de la Comunicación (Costa, 2001). Este autor se refiere a la comunicología explicándola como Comunicación aplicada, que llamamos y que es el puente estratégico entre la teoría y la praxis, entre la ciencia social y las ciencias empresariales.

Cabe señalar que en la mayoría de los textos de las especialidades de Economía y de Comunicación, se entrega información de empresas, pero no existe una bibliografía considerable en lo que respecta a la Cultura Organizacional en instituciones no lucrativas, es decir, organizaciones estatales o fundaciones.

En numerosas empresas de todo el mundo, y también en Chile, se están aplicando una serie de conceptos, como elementos constitutivos de una definición de cultura, los principales o los más difundidos, son los de visión, misión y valores. De forma sintética y poniendo el acento en lo referente a la RS y la Comunicación, se analizan estos conceptos.

La visión debe tener las siguientes características: ser imaginable y visualizable, deseable para los empleados, posible de conseguir, aunque no fácil, concreta y proporcionar claves a los directivos, flexible para permitir las iniciativas individuales y ser comunicable de forma sencilla y breve. Es decir, la visión es lo que «se propone ser» la institución o empresa. La misión, sirve para «humanizar» la estrategia de la empresa y en ella se deben ver reflejadas las respuestas de por qué y para qué existe esta organización y para qué trabajamos allí. Es el objetivo de la empresa, es lo que hay que hacer. En la misión deben estar incorporadas las políticas de comunicación externa. Los valores, por su parte, son los que definen el alma de una empresa. "Los valores son los ejes de conducta de una empresa y están relacionados con el propósito 
de la misma», puntualiza Cordón Portillo (2004). Los conceptos reseñados, definen y componen lo que es la Cultura de una organización y por tanto son elementos imprescindibles para considerar en todo plan de Comunicación Estratégica institucional y en la administración interna de la misma.

\subsection{Comunicación Organizacional Estratégica}

La Comunicación y los estudios que se realizaban sobre ella, permitieron que esta área del conocimiento se convirtiera en Ciencia alrededor de 1945, a partir de los trabajos y aportes multidisciplinarios, entre los que es necesario destacar los de Wiener y Shannon, considerados como los padres de esta no tan joven disciplina. Aunque los precitados estudios influyeron de manera importante en otras áreas del saber, es necesario señalar que desde la perspectiva de la Ciencia de la Comunicación, la revolución de mayor impacto a nivel mundial se da en la década de los 70 y 80, con la irrupción de la Comunicación Digital.

Y es por ello que en esta Era de la Información o Era del Conocimiento, es indispensable conocer y considerar la Comunicación Digital, como también saber hacer un uso adecuado, eficiente y eficaz de las Tecnologías de la Información y la Comunicación -TIC-. La afirmación es corroborada por cifras y estadísticas mundiales, como por ejemplo, que entre 2000 y 2017 el uso de la Internet en el mundo ha tenido un crecimiento de un $936 \%$, y desde el punto de vista de la penetración de Internet en el mundo, por continente, los porcentajes más altos se dan en Norteamérica con un $88,1 \%$; Europa 77,4\% y Oceanía/ Australia, 68,1\%. En un plano intermedio Latinoamérica 59,6\% y Oriente Medio $56,7 \%$ y el más bajo se da en África, con un 28,3\%.

Tabla 1. Estadísticas mundiales del internet y de la población

\begin{tabular}{|c|c|c|c|c|c|c|}
\hline Regiones & $\begin{array}{l}\text { Población } \\
\text { (2017 Est.) }\end{array}$ & $\begin{array}{l}\text { Usuarios } \\
\text { Dic. } 31 \\
(2000)\end{array}$ & $\begin{array}{c}\text { Usuarios } \\
\text { (Mar. } 31 \\
\text { 2017) }\end{array}$ & $\begin{array}{l}\text { Crecimien- } \\
\text { to } \% \\
(2000-2017)\end{array}$ & $\begin{array}{c}\text { Penetra- } \\
\text { ción (\% } \\
\text { Población) }\end{array}$ & $\begin{array}{c}\text { Facebook } \\
\text { Junio 30, } 2016\end{array}$ \\
\hline África & 1246504865 & 4514400 & 353121578 & $7722,1 \%$ & $28,3 \%$ & 146637000 \\
\hline Asia & 4148177672 & 114304000 & 1874136654 & $1539,6 \%$ & $45,2 \%$ & 559003000 \\
\hline Europa & 822710362 & 105096093 & 636971824 & $506,1 \%$ & $77,4 \%$ & 309576660 \\
\hline Oriente Medio & 250327574 & 3284800 & 141931765 & $4220,9 \%$ & $56,7 \%$ & 76000000 \\
\hline Norte América & 363224006 & 108096800 & 320068243 & $196,1 \%$ & $88,1 \%$ & 223081200 \\
\hline $\begin{array}{l}\text { Latinoamérica } \\
\text { / Caribe }\end{array}$ & 647604645 & 18068919 & 385919382 & $2035,8 \%$ & $59,6 \%$ & 296636180 \\
\hline $\begin{array}{l}\text { Oceanía / } \\
\text { Australia }\end{array}$ & 40479846 & 7620480 & 27549054 & $261,5 \%$ & $68,1 \%$ & 18239110 \\
\hline $\begin{array}{l}\text { TOTAL } \\
\text { MUNDIAL }\end{array}$ & 7519028970 & 360985492 & 3739698500 & $936,0 \%$ & $49,7 \%$ & 1679433530 \\
\hline
\end{tabular}

Fuente: Miniwatts Marketing Group (2017). 
Es pertinente agregar que además de las variables de desarrollo económico que allí se reflejan, existe un rango etario de la población que «no nació con el chip incorporado» ${ }^{1}$ (Rueda y Quintana, 2007) y para esas personas o públicos que no han recibido una alfabetización tecnológica, se requiere investigar, conocer, aplicar y difundir esta modalidad comunicacional, que en definitiva es más usada que conocida, es decir, las personas conocen más de sus herramientas, como Internet o Web, que de Comunicación Digital, como un área específica de estudio.

Internet poco a poco ha pasado de ser una enorme biblioteca de información a un nuevo medio de información y en algunos casos de comunicación, el cual ofrece atributos como credibilidad, inmediatez, complementación de servicios multimedia y actualización constante, algo de lo que carecen el resto de los medios tradicionales de comunicación, puesto que la nota que se escucha en la mañana en la TV o en la Radio, se repite en las emisiones posteriores.

Es necesario señalar, que entre las características de Internet se pueden destacar su instantaneidad, que permite en la entrega de información y en la relación de redes virtuales; la inter-textualidad, es decir, una nueva forma de relación con los textos, y la interactividad permanente. Por otra parte, el ciberperiodismo o periodismo digital, ha significado una nueva revolución dentro de la Web, la cual ha puesto a los periodistas ante el reto de tener a la mano un medio que exige la difusión de la información escrita de manera inmediata, en tiempo real, y con la ventaja de actualizarse varias veces al día. En definitiva, la Comunicación Digital tiene ventajas, pero también amenazas, especialmente desde la perspectiva del «nuevo concepto de velocidad» (Postigo-Gómez, 2009) o tiempo que se da con las TIC, nos referimos a la inmediatez en la entrega de información.

Estas nuevas herramientas tecnológicas de informar también han tenido su influencia en los diversos tipos de comunicación que se dan en la sociedad y en este caso en las instituciones o empresas. Sólo a manera de ejemplo, se señala que en una investigación, realizada en Europa el 2002, se detectó que «las Intranets son uno de los principales medios para la comunicación interna en las empresas, estando tan sólo por debajo de los House Organ» (Bell Mallén, 2004).La citada conclusión fue el producto de una investigación realizada por la Federación Europea de Comunicación Empresarial y el Departamento de Comunicación de la Universidad de Salzburgo, entidades que publicaron, a finales del 2002, un estudio delphi sobre la comunicación interna en empresas en Europa.

Comunicación Organizacional, Comunicación Institucional, Comunicación Corporativa, son algunos de los nombres que se dan a la Comunicación, tanto interna, como externa, en una organización. A ellos, que tienen la palabra Comunicación, habría que agregar, los conceptos de Imagen Corporativa, Identidad Corporativa, Clima Organizacional, Branding Corporativo y Responsabilidad Social, especialidades todas que tienen como punto de partida o finalidad la comunicación.

Que la comunicación es básica en toda organización, no hay duda, que como planteó Watzlawick (1981), «todo comunica», también. Sin embargo, aún la Comunicación, al igual como la RS, no han logrado ser masivamente valorados. Aunque existe abundan-

1 Nombre que Rueda y Quintana (2007) dan a los no-nativos digitales. 
te bibliografía sobre la comunicación, aún hay instituciones o empresas que no le dan la importancia que ella tiene para un manejo exitoso de toda organización.

Un claro ejemplo de la importancia de esta disciplina, se da, de manera evidente, en la Comunicación Estratégica, cuyo objetivo es el de «convertir los vínculos que las organizaciones tienen con sus entornos cultural, social y político, en una relación armónica y positiva, desde el punto de vista de sus intereses, en definitiva el propósito último, es convertir los vínculos en una ventaja competitiva» (Rojas, 2007). Una de las tareas fundamentales de la Comunicación Estratégica en una institución o empresa, es la de producir adhesión de parte, tanto de su público objetivo, como de su entorno relevante.

En definitiva se puede señalar que la comunicación, sea ésta de cualquiera especificidad, es un elemento imprescindible a considerar en todo tipo de organización, no importando la naturaleza jurídica o social que la defina y le de existencia legal.

\section{Discusión y conclusiones}

En la Introducción del presente artículo se señala como premisa que la Comunicación es una de las formas básicas o elementales que tiene la RS, es decir, toda institución, organización o empresa, debe comunicarse / informar con su entorno, y no hacerlo exclusivamente con una finalidad publicitaria o propagandística, es decir, para promocionar sus productos o servicios, sino que debe informar a los que están al lado, a los que la rodean, a su entorno, sobre quién es, qué hace, cómo lo hace y para qué lo hace.

También se planteó que la RS, el Entorno y la Comunicación, estaban unidos en su desarrollo y campo investigativo. Esta última, por cuanto se reitera que la información y la comunicación son una de las necesidades que deben ser satisfechas y contempladas por toda organización que desee ser socialmente responsable y con ello alcanzar el prestigio y reputación anhelados.

Desde la perspectiva de la Comunicación Digital, se considera que una organización es moderna, si cuenta con computadores -ordenadores- pero eso no basta y no lo es todo, es necesario capacitar a las personas en el uso de esas TIC y al mismo tiempo informar a los directivos, sobre las ventajas y amenazas que tienen esos instrumentos.

Aún falta mucho por hacer o más bien aún falta mucho por informar, en lo que respecta a la RS y el papel que le corresponde a la Comunicación en ese ámbito. Cuando se afirma que falta mucho por informar a la opinión pública, se parte de un hecho concreto y comprobable: en Chile sólo hace siete o diez años, se han realizado seminarios, encuentros y diversas actividades educativo-culturales sobre la RS, también han sido publicadas informaciones sobre RS. Sin embargo, desde fines de la década de los 80, en algunas universidades chilenas, comenzó a estudiarse la Teoría de la Responsabilidad Social, y se inició, en las Facultades y Carreras de Economía y de Ingeniería Comercial.

No se puede señalar que ese desconocimiento a nivel general sobre la RS se deba o sea de responsabilidad de los periodistas, porque ellos no son quienes fijan las pautas informativas de los diarios, radios y de la televisión. Tampoco sería justo decir que son los dueños de esos medios. La situación es más compleja y aquí y ahora no se podría entregar una respuesta, pero sí aportar al decir que en esta situación de desinformación, influyen muchos factores, los más evidentes son los diversos poderes eco- 
nómicos y socio-políticos del país, las políticas de comunicación de las universidades y los medios de comunicación.

Sin embargo, es destacable que en el momento actual, aunque sea tardíamente, se está difundiendo más ampliamente lo relativo a la RS, y se puede encontrar tanto, en el mundo real, como en el virtual, es decir, en Internet, desde bibliografía seria, científica, hasta organizaciones creadas justamente sobre la base de la RS. En Chile -por ejemplo- la Fundación Pro-humana (2017), ha realizado algunos aportes. A pesar de estos avances, aún persisten carencias, desinformación y algunos prejuicios. Se agrega que en Chile y en varios países de América Latina, la opinión pública, como la sociedad civil en general, han comenzado no tan sólo a esperar sino que a exigir que las organizaciones se comporten como «buenos ciudadanos corporativos». Para las empresas que operan en Chile esto ya no es sólo una condición ética sino un imperativo para el éxito de sus negocios en el largo plazo.

Por otra parte, se puntualiza, que existen pocos aportes de especialistas en las Ciencias Sociales en general y en particular en la Ciencia de la Comunicación, en el tema de la RS. Además, aunque hay avances importantes en el ámbito académico o de investigación en esa área, aún no se han difundido suficientemente, y es de esperar que pronto sean realizados.

Un ejemplo del desconocimiento y aplicación que existe, es el hecho que sólo algunas empresas e instituciones realizan auditorias en Comunicación, técnica bastante aplicada en organizaciones de países del primer mundo. Incluso algunos autores, van más allá de una auditoría comunicacional y la denominan «Auditoría Estratégica Global». Esta «Auditoría Estratégica Global» la presenta Costa (2001) al señalar que:

Es un hecho que el término auditoría, auditar, tiene connotaciones contables: designa una revisión de cuentas, cuyos datos ya son previamente conocidos por la empresa. Mi auditoría no consiste en revisar y comprobar datos que son bien conocidos a priori. Es todo lo contrario: una revelación de datos ignorados, pero que existen, están aquí e interactúan de algún modo con la empresa (p. 241).

Relacionado con la comunicación y la información, se agrega que la Ley de Transparencia y acceso a la Información Pública (Chile), tiene como principios la transparencia de la función pública, derecho de acceso a la información de los órganos de la Administración del Estado, procedimientos para el ejercicio del derecho y para su amparo y las excepciones a la publicidad de la información (Ley $\mathrm{N}^{\circ} 20.285$, 2008). Se cita este cuerpo legal porque allí está reflejado, aunque de manera impositiva, a través del mandato de la ley, uno de los principios aquí reiterados y es el de la obligación y la necesidad de informar la labor que llevan a cabo los organismos estatales. Esta misma transparencia la está requiriendo la sociedad en varios otros ámbitos, extendiéndose hasta la actividad privada.

Para futuras investigaciones y algunas ya en curso, la Comunicación continuará siendo un objeto de estudio de interés, y aquí se propone iniciar algunos sobre la Responsabilidad Individual, que estaría directamente relacionada con el tema de la Confianza y como Construir confianza (Enderle, 2003, pp.131-132). 


\section{Referencias}

Andrews, K.R. (1977). Concepto de Estrategia de la Empresa, edición en español. Pamplona: Ediciones Universitarias de Navarra S.A., EUNSA,

Beck, U. (2006). La sociedad del riesgo. Edición en español. Editorial Paidós, Primera Edición, Alemania, 1986.

Bell Mallén, J.I. (2004). Comunicar para crear valor. La Dirección de comunicación en las organizaciones. Universidad de Navarra, Ediciones Universidad de Navarra. EUNSA.

Biging (2018). Transporte urbano de Barcelona. (https://goo.gl/jtzz58) (23-06-2018).

Blog de Ética (2011) Definición de RSE: ya se está despejando el camino. https://goo.gl/4H6rTM (09-2011).

Cordón Portillo, A. (2004). La imagen de las empresas y su cultura. En Bell Mallén, J.I. (Ed.), Comunicar para crear valor. La Dirección de comunicación en las organizaciones (pp. 109-124). Ediciones Universidad de Navarra. EUNSA

Cortina, A., Sen, A., Castells, M., Conill, J., Ramonet, I., Dávila, A., Enderle, G., Cebrián, J.L., Villafañe, J., Schulte-Hillen, G., \& García-Marzá, D. (2003). Construir confianza. Ética de la empresa en la sociedad de la información y las comunicaciones. Madrid: Editorial Trotta, S.A.

Costa, J. (2001). Imagen corporativa en el siglo XXI. La Crujía Ediciones. Tucumán 1999, Argentina.

Capriotti, P.P. (2009). Branding Corporativo. Fundamentos para la gestión estratégica de la identidad Corporativa. Santiago, Chile: Andros Impresores.

Enderle, G. (2003). Competencia global y responsabilidad corporativa de las pequeñas y medianas empresas. En A. Cortina et al., Construir confianza. Ética de la empresa en la sociedad de la información y las comunicaciones. Madrid: Editorial Trotta, S.A.

Friedman, M. (1975). Bases de un desarrollo económico. Santiago de Chile: Fundación de Estudios Económicos.

Ley $N^{\circ}$ 20.285. (2008). Ley Sobre acceso a la información pública. Diario Oficial de la República de Chile, Santiago, Chile, 31 de agosto de 2008.

Miniwatts Marketing Group (2017). (https://goo.gl/36i3ZF) (23-04-2018).

Navarro, F. (2012). Responsabilidad social corporativa: teoría y práctica. Madrid, España: ESIC Editorial.

Paladino, M., Milberg, A., \& Sánchez, F. (2006). Emprendedores sociales $\&$ empresarios responsables. Buenos Aires, Argentina: Temas Grupo Editorial.

Porter, M., \& Kramer, M. (2006). Estrategia y sociedad. El vínculo entre la ventaja competitiva y la responsabilidad social competitiva. Harvard Business Review: América Latina, 84(12), 45-56. (https://goo.gl/2v6UKW).

Porter, M.E. (1991). La ventaja competitiva de las Naciones. Barcelona: Plaza \& Janes Editores S.A.

Porter, M.E. (2007). La ventaja competitiva de las Naciones. Harvard Business Review América Latina, 85(11), 69-95. (https://goo.gl/1DSA3e).

Postigo- Gómez, I. (2009). Módulo: Intranet como herramienta de la Comunicación Interna en la Organización. España: Universidad de Málaga.

Prohumana (2017). Prohumana Organización chilena sin fines de lucro. (https://goo.gl/8pJkzK) (30-09-2017).

Rochlin, S. (2005). Llevar la responsabilidad corporativa al ADN de su empresa. Harvard Business Review América Latina, 83(8), 26-35. (https://goo.gl/8pJkzK).

Rojas, V. (2007). Comunicación organizacional, corporativa y estratégica: reflexión en torno a conceptos y diferencias. Impreso El Mercurio de Valparaíso.

Rueda, O.R., \&, Quintana, R.A. (2007). Ellos vienen con el chip incorporado. Editoriales Universitarias de Colombia.

Sen, A., \& Kliksberg, B. (2007). Primero la gente. Una mirada desde la ética del desarrollo a los principales problemas del mundo globalizado. Barcelona: Ediciones Deusto.

Villafañe, J. (2003). Influencia de la Comunicación en la reputación corporativa. En A. Cortina et al., Construir confianza. Ética de la empresa en la sociedad de la información y las comunicaciones. Madrid: Editorial Trotta, S.A.

Watzlawick, P., Helmick, J., \& Jackson, D. (1981). Teoría de la Comunicación Humana. Barcelona, España: Editorial Herder. 